\title{
SOME RESULTS ON SYMMETRICALLY-PRESENTED GROUPS
}

\author{
by D. L. JOHNSON and R. W. K. ODONI
}

(Received 17th June 1992)

\begin{abstract}
Necessary and sufficient conditions are found on an ideal $a \triangleleft \mathbb{Z}[x]$ for the additive group $[a]+$ of $\mathbb{Z}[x] / a$ to be finite and cyclic. As a consequence, the abelianizations of certain cyclically-presented groups are computed explicitly.
\end{abstract}

1980 Mathematical subject classification: 20F05, 11 R09.

\section{Introduction}

Let $n \in \mathbb{N}$ and let $F_{n}$ be the free group on $n$ symbols $x_{1}, \ldots, x_{n}$. Let $S_{n}$ be the symmetric group on $\{1, \ldots, n\}$ and, for $\sigma \in S_{n}$, consider the automorphism of $F_{n}$ given by $x_{i} \mapsto x_{\sigma(i)}, 1 \leqq i \leqq n$, which we again denote by $\sigma$. Now for any word $w \in F_{n}$ and any subgroup $\Gamma \leqq S_{n}$, we define a group $G_{n}(w, \Gamma)$ by the presentation

$$
G_{n}(w, \Gamma)=\left\langle x_{1}, \ldots, x_{n} \mid \sigma(w), \sigma \in \Gamma\right\rangle=F_{n} / N,
$$

where $N$ is the normal closure of $\{\sigma(w): \sigma \in \Gamma\}$ in $F_{n}$, and call groups of this type symmetrically presented.

The chief aims of this paper are to provide criteria for the abelianization $G_{n}(w, \Gamma)^{a b}$ of $G_{n}(w, \Gamma)$ to be finite and finite cyclic, respectively. We remark that special cases of $G_{n}(w, \Gamma)$ have been discussed by numerous authors, and cite as examples (all with $\Gamma=\langle(12 \ldots n)\rangle)$ :

(i) the 3-manifold groups [9]

$$
K_{n}, \quad w=x_{1} x_{3} x_{2}^{-1}
$$

(ii) the Fibonacci groups of Conway $[3,4,8]$

$$
F(r, n), \quad w=x_{1} x_{2} \ldots x_{r} x_{r+1}^{-1},
$$

(iii) and their generalizations

$$
F(r, s, c, n), \quad w=x_{1} x_{2} \ldots x_{r} x_{s+1}^{-c}
$$


where all subscripts are reduced modulo $n$ to lie in the set $\{1,2, \ldots, n\}$. (For the special case $c=1$ see [2]).

For $u \in F_{n}$, let $\bar{u}$ denote the image of $u$ in $F_{n}^{a b}$, so that $F_{n}^{a b}$ is the free abelian group on $\bar{x}_{1}, \ldots, \bar{x}_{n}$. Denoting the binary operation in $F_{n}^{a b}$ by + , we have that $F_{n}^{a b} \cong \mathbb{Z}^{n}$ as a $\mathbb{Z}$-module. Moreover, $F_{n}^{a b}$ has an obvious (left) $\mathbb{Z} \Gamma$-module structure induced by the permutation action of $\Gamma$ on $x_{1}, \ldots, x_{n}$, and then

$$
G_{n}(w, \Gamma)^{a b} \cong F_{n}^{a b} / \mathbb{Z} \Gamma \cdot \bar{w} .
$$

We give in Section 1 some general criteria for the finiteness of $G_{n}(w, \Gamma)^{a b}$ based on the isomorphism (0.5). Roughly speaking, there is a sharp dichotomy between the cases $\Gamma$ transitive and $\Gamma$ intransitive. Specifically, we show that when $\Gamma$ is intransitive $G_{n}(w, \Gamma)^{a b}$ is infinite for every $w \in F_{n}$, while in the transitive case $G_{n}(w, \Gamma)^{a b}$ is finite for "almost all" $w \in F_{n}$. In Section 2 , we consider the case where $\Gamma$ is transitive abelian, and show that the structure of $G_{n}(w, \Gamma)^{a b}$ is that of the additive group $[a]_{+}$of $\mathbb{Z}\left[t_{1}, \ldots, t_{k}\right] / \mathfrak{a}$ for some ideal $a$ in the polynomial ring $\mathbb{Z}\left[t_{1}, \ldots, t_{k}\right]$ in $k$ (commuting) independent variables $t_{1}, \ldots, t_{k}$ over $\mathbb{Z}$. In Section 3 we give a simple necessary and sufficient condition for $[a]_{+}$to be finite cyclic, while in Section 4 we illustrate our results in the case where $\Gamma$ is cyclic with specific calculations for examples $(0.2)$ and $(0.4)$ above.

We are indebted to several people for useful discussions and criticisms during the course of writing this paper. In particular, we acknowledge the contributions of $\mathrm{K}$. A. Brown, M. W. Bunder, A.-C. Kim, S. J. Pride, G. C. Smith and M. J. Tomkinson.

\section{Finiteness criteria}

Throughout this section, " $R$-module" will mean "left $R$-module". Various standard results from representation theory will be quoted; for these [5] is a convenient reference.

We start from the isomorphism (0.5), and for convenience identify $\bar{x}_{i}$ with the row vector $(0, \ldots, 0,1,0, \ldots, 0)$, having 1 in the $i$ th column, in $\mathbb{Z}^{n}$. Let $E=\sum_{i=1}^{n} \mathbb{Z} \bar{x}_{i}$, and for subrings $R$ of $\mathbb{C}$ let $R \cdot E=\sum_{i=1}^{n} R \bar{x}_{i}$; this has a natural $R \Gamma$-module structure induced by the action of $\Gamma$ on $\left\{x_{1}, \ldots, x_{n}\right\}$.

Lemma 1.1. (i) $G_{n}(w, \Gamma)^{a b}$ is finite if and only if $\mathbb{Q} \cdot E=\mathbb{Q} \Gamma \cdot \bar{w}$.

(ii) $\mathbb{Q} \cdot E=\mathbb{Q} \Gamma \cdot \bar{w}$ implies that $\mathbb{C} \cdot E=\mathbb{C} \Gamma \cdot \bar{w}$.

Proof. Of these, only (i) requires proof. Suppose first that $G_{n}(w, \Gamma)^{a b}$ is finite, of order $k$ say. Then $k \bar{x}_{i} \in \mathbb{Z} \Gamma \cdot \bar{w}$ for $i \leqq n$, so that $\bar{x}_{1}, \ldots, \bar{x}_{n} \in \mathbb{Q} \Gamma \cdot \bar{w}$, whence $\mathbb{Q} \cdot E=\mathbb{Q} \Gamma \cdot \bar{w}$. For the converse, if $\mathbb{Q} \cdot E=\mathbb{Q} \Gamma \cdot \bar{w}$, then $\bar{x}_{i}=\lambda_{i} \bar{w}$ with $\lambda_{i} \in \mathbb{Q} \Gamma, 1 \leqq i \leqq n$. Choosing $d \in \mathbb{N}$ such that $d \lambda_{1}, \ldots, d \lambda_{n} \in \mathbb{Z} \Gamma$, we see that $G_{n}(w, \Gamma)^{a b}$ is finite, of order dividing $d^{n}$.

Lemma. 1.2. If $\Gamma$ is intransitive, then $G_{n}(w, \Gamma)^{a b}$ is infinite for all $w \in F_{n}$. 
Proof. In view of Lemma 1.1 , it is enough to show that $\mathbb{C} \cdot E$ is not a cyclic $\mathbb{C} \Gamma$ module, that is, not of the form $\mathbb{C} \Gamma \cdot \xi$ for $\xi \in \mathbb{C} \cdot E$.

Suppose for a contradiction that $\Gamma$ is intransitive but $\mathbb{C} \cdot E$ is cyclic, so that there is a $\mathbb{C} \Gamma$-epimorphism $\pi: \mathbb{C} \Gamma \rightarrow \mathbb{C} \cdot E$. It follows that

$$
1=\left(\chi_{1}, \chi_{r}\right) \geqq\left(\chi_{1}, \chi_{e}\right),
$$

where $\chi_{r}, \chi_{e}, \chi_{1}$ are the characters of $\mathbb{C} \Gamma, \mathbb{C} \cdot E$, and the trivial $\mathbb{C} \Gamma$-module, respectively. But $\left(\chi_{1}, \chi_{e}\right)$ is just the number of orbits of $\Gamma$ on $\{1,2, \ldots, n\}$, and this is greater than 1 by hypothesis.

We assume henceforth that $\Gamma$ is transitive and fix the following notation:

$$
\begin{gathered}
H=\operatorname{stab}\left(x_{1}\right), \quad \Gamma=\bigcup_{i=1}^{n} \gamma_{i} H, \\
e_{H}=|H|^{-1} \sum_{h \in H} h \in \mathbb{Q} \Gamma,\left(e_{H}^{2}=e_{H}\right) .
\end{gathered}
$$

Lemma 1.3. For transitive $\Gamma, \mathbb{Q} \cdot E \cong \mathbb{Q} \Gamma \cdot e_{H}$ and so $\mathbb{Q} \cdot E$ is a cyclic $\mathbb{Q} \Gamma$-module.

Lemma 1.4. Let $\Gamma$ be transitive and put $M=\mathbb{Q} \Gamma \cdot e_{H}$. Then (i) the elements $v_{i}=\gamma_{i} e_{H}$ form $a \mathbb{Q}$-basis for $M$, and (ii) there is a non-zero homogeneous polynomial $P_{\Gamma}=$ $P_{\Gamma}\left(t_{1}, \ldots, t_{n}\right) \in \mathbb{Q}\left[t_{1}, \ldots, t_{n}\right]$ of degree $n$ in $n$ commuting indeterminates such that, for $q_{i} \in \mathbb{Q}$, $1 \leqq i \leqq n, \mathbb{Q} \Gamma \cdot \sum_{i=1}^{n} q_{i} v_{i}=M$ whenever $P_{\Gamma}\left(q_{1}, \ldots, q_{n}\right) \neq 0$.

Proof. Part (i) is clear. For (ii), let $L(\mu): M \rightarrow M$ be the $\mathbb{Q}$-linear map sending $v_{i}$ to $v_{i} \mu, 1 \leqq i \leqq n, \mu \in M$. For $q_{1}, \ldots, q_{n} \in \mathbb{Q}$, we have

$$
L\left(\sum_{i=1}^{n} q_{i} v_{i}\right)=\sum_{i=1}^{n} q_{i} L\left(v_{i}\right)
$$

Now define

$$
P_{\mathrm{I}}\left(t_{1}, \ldots, t_{n}\right)=\operatorname{det}\left(\sum_{i=1}^{n} t_{i} L\left(v_{i}\right)\right) \in \mathbb{Q}\left[t_{1}, \ldots, t_{n}\right]
$$

Then

$$
\operatorname{det}\left(\sum_{i=1}^{n} q_{i} L\left(v_{i}\right)\right)=\operatorname{det}\left(L\left(\sum_{i=1}^{n} q_{i} v_{i}\right)\right)=P_{\Gamma}\left(q_{1}, \ldots, q_{n}\right)
$$

whenever $q_{1}, \ldots, q_{n} \in \mathbb{Q}$. Clearly $\operatorname{det}\left(L\left(e_{H}\right)\right)=1$, so that $P_{\Gamma} \neq 0$ in $\mathbb{Q}\left[t_{1}, \ldots, t_{n}\right]$. Let 
$q_{1}, \ldots, q_{n} \in \mathbb{Q}$ with $P_{\Gamma}\left(q_{1}, \ldots, q_{n}\right) \neq 0$ and set $\mu=\sum_{i=1}^{n} q_{i} v_{i}$. Then $\operatorname{det}(L(\mu)) \neq 0$ so that $L(\mu)$ is surjective and $M=M \mu$. Hence

$$
\mathbb{Q} \Gamma \cdot \mu \subseteq M=M \mu \subseteq \mathbb{Q} \Gamma \cdot \mu
$$

that is, $M=\mathbb{Q} \Gamma \cdot \mu$ as required. That $P_{\Gamma}$ is homogeneous of degree $n$ is clear from the definition.

Corollary 1.5. For transitive $\Gamma$, there is a non-zero homogeneous polynomial $P_{\Gamma}^{*}$ of degree $n$ in $n$ variables such that $G_{n}(w, \Gamma)^{a b}$ is finite whenever $P_{\Gamma}^{*}\left(r_{1}, \ldots, r_{n}\right) \neq 0$, where $\bar{w}=\sum_{i=1}^{n} r_{i} \bar{x}_{i}$.

We remark that, in any given case of interest, there is in principle no difficulty in calculating the polynomial $P_{\Gamma}^{*}$. Indeed, taking $\bar{x}_{i}$ as $\gamma_{i} e_{H}$ in $\mathbb{Q} \Gamma \cdot e_{H}$, where $\gamma_{i} x_{1}=x_{i}, P_{\Gamma}^{*}$ is just the $P_{\Gamma}$ of Lemma 1.4 .

\section{Transitive abelian $\Gamma$}

The results of the previous section can be greatly refined and simplified in the favourable special case when $\Gamma$ is transitive and abelian, and we make this assumption now.

In this case, it is clear that $H=\{1\},|\Gamma|=n$, and the action of $\Gamma$ on $\left\{x_{1}, \ldots, x_{n}\right\}$ is equivalent to the (left) regular representation of $\Gamma$ on itself. We can thus index the $x$ 's by the elements of $\Gamma$, so that

$$
\gamma x_{\delta}=x_{\gamma \delta}, \text { for all } \gamma, \delta \in \Gamma
$$

and $E \cong \mathbb{Z} \Gamma$ as $\mathbb{Z} \Gamma$-modules. Now write

$$
\bar{w}=\sum_{\gamma \in \Gamma} r(\gamma) \bar{x}_{\gamma} \in G_{n}(w, \Gamma)^{a b}
$$

and associate with $w$ the element

$$
w^{*}=\sum_{\gamma \in \Gamma} r(\gamma) \gamma \in \mathbb{Z} \Gamma
$$

As $E \cong \mathbb{Z} \Gamma$, our isomorphism (0.5) can in this case be expressed as

$$
G_{n}(w, \Gamma)^{a b} \cong \mathbb{Z} \Gamma / \mathbb{Z} \Gamma \cdot w^{*}
$$

In the first place, $(2.1)$ yields a simple formula for the order of $G_{n}(w, \Gamma)^{a b}$. For $\alpha \in \mathbb{Q} \Gamma$, let $L_{\alpha}$ be the $\mathbb{Q}$-linear map from $\mathbb{Q} \Gamma$ to itself sending $\gamma \in \Gamma$ to $\alpha \gamma$, and let $N(\alpha)$ be the determinant of $L_{\alpha}$. It is clear that $N(\alpha) \in \mathbb{Q}$ and is non-zero if and only if $\alpha$ is a unit in 
$\mathbb{Q} \Gamma$. We can evaluate $N(\alpha)$ using the characters of $\Gamma$, as follows. Let $\Gamma^{*}=\operatorname{Hom}\left(\Gamma, \mathbb{C}^{*}\right)$ be the character group of $\Gamma$. For $\chi \in \Gamma^{*}$, we extend $\chi$ to a $\mathbb{Q}$-algebra homomorphism from $\mathbb{Q} \Gamma$ to $\mathbb{C}$ by defining

$$
\chi\left(\sum_{\gamma \in \Gamma} a_{y} \gamma\right)=\sum_{\gamma \in \Gamma} \alpha_{\nu} \chi(\gamma), \text { all } a_{\gamma} \in \mathbb{Q} .
$$

Then it is easily checked that

$$
N(\alpha)=\prod_{x \in \Gamma^{*}} \chi(\alpha)
$$

Now consider (2.1). If $N\left(w^{*}\right)=0$, then $\mathbb{Q} \Gamma \cdot w^{*} \neq \mathbb{Q} \Gamma=E$, and so $G_{n}(w, \Gamma)^{a b}$ is infinite; otherwise, $N\left(w^{*}\right)$ is a non-zero integer (since it is an algebraic integer in $\mathbb{Q}$ ), and $\left|N\left(w^{*}\right)\right|$ is the order of the (finite) group $G_{n}(w, \Gamma)^{a b}$, which can thus be evaluated using (2.2).

These calculations do not, of course, say anything about the structure of $G_{n}(w, \Gamma)^{a b}$. For this purpose, it is generally more convenient to present $\mathbb{Z} \Gamma$ as a quotient of a polynomial ring over $\mathbb{Z}$. To do this, suppose that $\Gamma$ is the direct product of $k$ finite cyclic groups of orders $n_{1}, \ldots, n_{k}$, so that $n=\prod_{i=1}^{k} n_{i}$. Let $t_{1}, \ldots, t_{k}$ be independent (commuting) variables over $\mathbb{Z}$. Then we clearly have a ring epimorphism

$$
\pi: \mathbb{Z}\left[t_{1}, \ldots, t_{k}\right] \rightarrow \mathbb{Z} \Gamma
$$

with $\operatorname{Ker} \pi=\left(t_{1}^{n_{1}}-1, \ldots, t_{k}^{n_{k}}-1\right)$. Choosing $f \in \mathbb{Z}\left[t_{1}, \ldots, t_{k}\right]$ such that $\pi(f)=w^{*}$, it follows from (2.1) that

$$
G_{n}(w, \Gamma)^{a b} \cong \mathbb{Z}\left[t_{1}, \ldots, t_{k}\right] / \mathfrak{a},
$$

where

$$
\mathfrak{a}=\left(t_{1}^{n_{1}}-1, \ldots, t_{k}^{n_{k}}-1, f\right) \text {. }
$$

This leads to the following general problem: if $R=\mathbb{Z}\left[t_{1}, \ldots, t_{k}\right]$ and $a \triangleleft R$, then what is the structure of $[a]_{+}$, the additive group of $R / a$ ? In the next section, we find simple necessary and sufficient conditions for $[a]_{+}$to be finite cyclic.

\section{Cyclic quotients of $\mathbb{Z}\left[t_{1}, \ldots, t_{k}\right]$}

This section will be devoted to proving the following theorem, which is the main result of this paper.

Theorem 3.1. Let $k \in \mathbb{N}, R=\mathbb{Z}\left[t_{1}, \ldots, t_{k}\right]$, and $\mathfrak{a} \triangleleft R$. Then the additive group $[a]_{+}$of $R / \mathfrak{a}$ is finite and cyclic if and only if the following two conditions hold. 
(i) $a \cap \mathbb{Z} \neq 0$, and

(ii) for all primes $p \in \mathbb{N}$, either $a+p R=R$ or $\mathfrak{a}+p R=\left(p, t_{1}-a_{1}, \ldots, t_{k}-a_{k}\right)$ for some $\left(a_{1}, \ldots, a_{k}\right) \in \mathbb{Z}^{k}$ depending on $p$.

Proof. We first prove necessity. Suppose that $[a]_{+}$is cyclic of order $v \in \mathbb{N}$. Then $v R \subseteq a$, so that $0 \neq v \mathbb{Z} \subseteq a \cap \mathbb{Z}$ and (i) holds. Now let $p \in \mathbb{N}$ be prime. Then $[a+p R]_{+}$is a quotient of $[\mathfrak{a}]_{+}$and is thus finite and cyclic. Suppose that $\mathfrak{a}=\left(f_{1}, \ldots, f_{s}\right)$ and let $f_{i}$ be the $\bmod p$-reduction of $f_{i}, 1 \leqq i \leqq s$. There is a ring isomorphism

$$
R / \mathfrak{a}+p R \cong \mathbb{F}_{p}[t] /\left(\bar{f}_{1}, \ldots, \bar{f}_{s}\right)
$$

where $\mathbb{F}_{p}$ is the field $\mathbb{Z} / p \mathbb{Z}$ and $t=\left(t_{1}, \ldots, t_{k}\right)$, induced by reduction modulo $p$. Since the right-hand side of (3.1) is an $\mathbb{F}_{p}$-algebra, its additive group has exponent $p$ or 1 . Thus, $R / \mathfrak{a}+p R$ is either 0 or $\mathbb{F}_{p}$, that is, $a+p R$ is equal either to $R$ or to $\left(p, t_{1}-a_{1}, \ldots, t_{k}-a_{k}\right)$ for some $\left(a_{1}, \ldots, a_{k}\right) \in \mathbb{Z}^{k}$. Thus, (ii) holds.

The proof of sufficiency is rather harder. We assume that (i) and (ii) both hold, with $a \cap \mathbb{Z}=m \mathbb{Z}$ say, $m \in \mathbb{N}$, so that $m R \subseteq \mathfrak{a}$. We factorise $m=\prod_{j} p_{j}^{e_{j}}$ with distinct primes $p_{j} \in \mathbb{N}$, and all $e_{j} \in \mathbb{N}$, and put $\mathfrak{b}_{j}=\mathfrak{a}+p_{j}^{e_{j}} R$. Then $\mathfrak{b}_{i}+\mathfrak{b}_{j}=R$ for $i \neq j$, while

$$
\mathfrak{a} \subseteq \bigcap_{j} \mathfrak{b}_{j}=\prod_{j} \mathfrak{b}_{j} \subseteq \mathfrak{a}+m R=\mathfrak{a}
$$

Hence, $\mathfrak{a}=\prod_{j} \mathfrak{b}_{j}$. The Chinese remainder theorem now gives

$$
R / \mathfrak{a} \cong \prod_{j} R / \mathfrak{b}_{j}
$$

and

$$
[a]_{+}=\bigoplus_{j}\left[b_{j}\right]_{+} \cdot
$$

Since $p_{j}^{e_{j}} R \subseteq \mathrm{b}_{j},\left[\mathrm{~b}_{j}\right]_{+}$is a $p_{j}$-torsion group (possibly trivial), so that $[\mathrm{a}]_{+}$is finite cyclic if and only if each $\left[b_{j}\right]_{+}$is. We now show that condition (ii) guarantees the latter.

Let $p$ be any one of the $p_{j}$, and let $e=e_{j} \geqq 1$ and $\mathfrak{b}=\mathfrak{a}+p^{e} R=b_{j}$. First of all we must have $a+p R \neq R$. For otherwise we can solve $1=a+p r, a \in \mathfrak{a}, r \in R$, and then $p^{-1} m=$ $p^{-1} m a+m r \in \mathfrak{a}$ since $m \in \mathfrak{a}$, which contradicts $\mathfrak{a} \cap \mathbb{Z}=m \mathbb{Z}$. We thus have

$$
a+p R=\left(p, t_{1}-a_{1}, \ldots, t_{k}-a_{k}\right)
$$

for some $\mathbf{a}=\left(a_{1}, \ldots, a_{k}\right) \in \mathbb{Z}^{k}$. We now approach the core of the proof, which is embodied in the following assertion.

$\left(^{*}\right)$ For each $n \in \mathbb{N}$, there is a corresponding $\mathbf{b}=\mathbf{b}(n) \equiv \mathbf{a}(\bmod p)$ in $\mathbb{Z}^{k}$ such that $\left(p^{n}, t_{1}-b_{1}, \ldots, t_{k}-b_{k}\right) \subseteq a+p^{n} R$. 
Let us first see how this yields the result. Letting $c=\left(p^{n}, t_{1}-b_{1}, \ldots, t_{k}-b_{k}\right)$, we see that $\left[a+p^{n} R\right]_{+}$is a quotient of $[c]_{+}$, and since $R / c \cong \mathbb{Z} / p^{n} \mathbb{Z}$, it follows that $[c]_{+}$is cyclic of order $p^{n}$. Taking $n=e=e_{j}, p=p_{j}$, we deduce that $\left[b_{j}\right]_{+}$is cyclic of order dividing $p_{j}^{e_{j}}$, and then the result follows from (3.3).

It remains to prove assertion ( $\left.{ }^{*}\right)$. This is done by induction on $n$, taking $\mathbf{b}(1)=\mathbf{a} \in \mathbb{Z}^{k}$ when $n=1$. Now let $n \geqq 1$ and suppose we have some $\mathbf{b} \equiv \mathbf{a}(\bmod p)$ in $\mathbb{Z}^{k}$ such that $\left(p^{n}, t_{1}-b_{1}, \ldots, t_{k}-b_{k}\right) \subseteq \mathfrak{a}+p^{n} R$. By applying a suitable $\mathbb{Z}$-automorphism of $R$, we see that without loss of generality we may take $b=0 \equiv \mathbf{a}(\bmod p)$, in which case,

$$
\mathfrak{a}+p R=\left(p, t_{1}, \ldots, t_{k}\right) \supseteq \mathfrak{a}+p^{n} R \supseteq\left(p^{n}, t_{1}, \ldots, t_{k}\right) .
$$

Now let $\mathfrak{a}=\left(f_{1}, \ldots, f_{s}\right), f_{i} \in R$, and let $\mathfrak{b}$ be the ideal $\left(t_{1}, \ldots, t_{k}\right)$ in $R$. Then there exist $h_{i}, g_{i j} \in R$ such that

$$
f_{i}=p h_{i}+\sum_{j=1}^{k} g_{i j} t_{j}, \quad 1 \leqq i \leqq s
$$

In particular,

$$
f_{i} \equiv \phi_{i}\left(\bmod \left(p, \mathbf{b}^{2}\right)\right), \quad 1 \leqq i \leqq s
$$

where $\phi_{i}=\sum_{j \leqq k} g_{i j}(0) t_{j}$; here $\theta(0) \in \mathbb{Z}$ means the evaluation of $\theta \in R$ at $\mathbf{t}=\mathbf{0}$. Clearly,

$$
\mathfrak{a}+p R=(p, \mathfrak{b})=\left(p, \phi_{1}, \ldots, \phi_{s}, \mathbf{b}^{2}\right)
$$

A simple calculation involving the comparison of polynomials of given total degree now shows that

$$
t_{q} \equiv \sum_{i=1}^{s} e_{q i} \phi_{i}(\bmod p), \quad 1 \leqq q \leqq k
$$

for some $e_{q i} \in \mathbb{Z}$. Let $\mathbf{E}$ be the $k \times s$ matrix $\left(e_{q i}\right)$ and $\mathbf{G}$ the $s \times k$ matrix $\left(g_{i j}(\mathbf{0})\right)$. Then (3.7) implies that

$$
\mathbf{E G}=\mathbf{I}+p \mathbf{V},
$$

where $\mathbf{I}$ is the $k \times k$ identity matrix and $\mathbf{V}$ is some $k \times k$ matrix over $\mathbb{Z}$, since the $t_{q}$ are (algebraically) independent modulo $p$.

Now define, for $1 \leqq q \leqq k, \psi_{q}=\sum_{i=1}^{s} e_{q i} f_{i} \in \mathfrak{a}$. It follows from (3.4) that

$$
\psi_{q}=p \sum_{i=1}^{s} e_{q i} h_{i}+\sum_{i=1}^{s} \sum_{j=1}^{k} e_{q i} g_{i j}(0) t_{j}+\rho_{q},
$$


where $\rho_{q} \in b^{2}$. Now, by hypothesis, $b \subseteq a+p^{n} R$, so that $a, p b, b^{2} \subseteq a+p^{n+1} R$. These facts, together with (3.8) and (3.9), imply that

$$
t_{q}+p \sum_{i=1}^{s} e_{q i} h_{i}(0) \in \mathbf{a}+p^{n+1} R, \quad 1 \leqq q \leqq k
$$

We now take $b_{q}^{\prime}=-p \sum_{i=1}^{s} e_{q i} h_{i}(0) \in p \mathbb{Z}, 1 \leqq q \leqq k$, and deduce that $p^{n+1}, t_{1}-b_{1}^{\prime}, \ldots, t_{k}-$ $b_{k}^{\prime} \in \mathfrak{a}+p^{n+1} R$, where $\mathbf{b}^{\prime} \equiv \mathbf{a} \equiv \mathbf{0}(\bmod p)$ in $\mathbb{Z}^{k}$. This completes the proof of assertion $\left({ }^{*}\right)$.

\section{Cyclic $\Gamma$ : two examples}

In the special case of cyclic $\Gamma$, which is the most frequently encountered in practice, it seems natural to call $G_{n}(w, \Gamma)$ cyclically presented. For the examples mentioned in the introduction, we shall find necessary and sufficient conditions on the parameters for $F(r, s, c, n)^{a b}$ to be finite, and describe the structure of $K_{n}^{a b}$ precisely. The $F(r, n)$ have been extensively studied, and their abelianizations form the subject of [6], [1], [7]; indeed the first of these contains a proof of part (i) of the following result for these groups.

Proposition 4.1. Let $\Gamma=\langle(12 \ldots n)\rangle, w \in F_{n}$, and $f(t)$ the exponent-sum polynomial of (2.4). Then

(i) $G_{n}(w, \Gamma)^{a b}$ is finite if and only if the resultant $\rho=f * g \neq 0$, where $g(t)=t^{n}-1$, and then its order is $|\rho|$, and

(ii) $G_{n}(w, \Gamma)^{a b}$ is finite cyclic if and only if in addition the highest common factor $(\bar{f}, \bar{g})$ in $\mathbb{F}_{p}[t]$ is linear for every prime $p$ dividing $|\rho|$.

Proof. (i) A relation matrix for $G_{n}(w, \Gamma)^{a b}$ is the circulant matrix $C$ whose first row consists of the exponent sums $e_{i}$ of $x_{i}$ in $w, 1 \leqq i \leqq n$. Then the determinant of $C$ is just the product of the values of $f(x)$ on all $n$th roots of unity, that is, $\pm \rho$. Thus, the group is infinite if and only if $\rho=0$ and otherwise has order $|\rho|$.

(ii) We assume that $\rho \neq 0$ and refer to the conditions of Theorem 3.1 with $a=(f, g)$. Since $\rho \neq 0$, (i) is automatic and (ii) splits into two cases.

(a) $p^{\prime}|\rho| \Leftrightarrow(\bar{f}, \bar{g})=1$ in $\mathbb{F}_{p} \Leftrightarrow a+p R=R$, where $R=\mathbb{Z}[t]$.

(b) $p|| \rho \mid \Leftrightarrow(\bar{f}, \bar{g})=(t-a) \Leftrightarrow a+p R=(p, t-a)$.

For our first example, consider the group $F(r, s, c, n)$ given by $(0.4)$ with associated polynomial

$$
f(t)=1+t+\cdots+t^{-1}-c t^{s}
$$

where $r, s, c \in \mathbb{Z}, r \geqq 2$. We seek necessary and sufficient conditions on the parameters for 
this to vanish on an $n$th root of unity and consider first four cases depending on the value of $c$.

Case (i): $c=r$. Then $f(1)=0$.

Case (ii): $c=0$ and $(r, n)=h>1$. Then $f$ vanishes on a primitive $h$ th root of unity.

Case (iii): $c= \pm 1, \exists m \mid n, m>1$, with $r \equiv 1(\bmod m)$ and either $s \equiv 0(\bmod m)$ and $c=1$, or $s \equiv m / 2(\bmod m)$ and $c=-1$, with $m$ even in the second case. Then $f$ vanishes on a primitive $m$ th root of unity.

Case (iv): $c=(-1)^{u} 2, s=1+3 u, r \equiv 3(\bmod 6)$ and $n \equiv 0(\bmod 6)$. If $\lambda$ is a primitive sixth root on unity, then

$$
f(\lambda)=1+\lambda+\lambda^{2}-(-1)^{u} \cdot 2 \cdot \lambda \cdot \lambda^{3 u}=1-\lambda+\lambda^{2}=0 .
$$

Proposition 4.2. The group $F(r, s, c, n)^{a b}$ is infinite in the above four cases and finite otherwise.

Proof. Only the second assertion requires proof, so we assume that $c \neq r$ (case (i)). If $c=0$ and $r, n$ are coprime, then $g_{*} f \neq 0$ and the group is finite. Thus, we may also assume that $c \neq 0$ (case (ii)). Now let $\lambda$ be a primitive $m$ th root of unity with $m \mid n$ and $f(\lambda)=0$. Then we claim that the parameters satisfy the conditions in case (iii) or case (iv). From $f(\lambda)=0$ we obtain

$$
\lambda^{r}-1=c \lambda^{s}(\lambda-1)
$$

whence,

$$
c \lambda^{s}-1=\lambda^{r}\left(c \lambda^{s-r+1}-1\right) .
$$

Since $|\lambda|=1,\left|c \lambda^{s}-1\right|=\left|c \lambda^{s-r+1}-1\right|$, and since $c \neq 0$ we have

$$
\lambda^{s-r+1}=\lambda^{ \pm s}
$$

If $\lambda^{s-r+1}=\lambda^{s}$, then $\lambda^{r-1}=1$ and it follows from (4.2) that

$$
(\lambda-1)\left(c \lambda^{s}-1\right)=0 .
$$

But $f(1) \neq 0$ so $\lambda \neq 1$ and $c \lambda^{s}=1$. This implies that $c= \pm 1=\lambda^{s}$, which gives case (iii).

There remains the possibility that $\lambda^{s-r+1}=\lambda^{-s}$, which we have to reduce to case (iv) under the assumption that $c \neq \pm 1$. Thus, $\lambda^{r-1}=\lambda^{2 s}$ and

$$
\frac{\lambda^{2 s+1}-1}{\lambda-1}=c \lambda^{s}
$$

using (4.1), with $c \notin\{0, \pm 1, r\}$ and $m=$ ord $\lambda>1$. Let ord $\lambda^{2 s+1}=d \mid m$. Taking norms from $Q(\lambda)$ to $Q$ in (4.3) we have 


$$
c^{\phi(m)}(-1)^{s \phi(m)}=\left(\Phi_{d}(1)\right)^{\phi(m) / \phi(d)} / \Phi_{m}(1)
$$

where $\Phi_{m}$ is the $m$ th cyclotomic polynomial and $\phi$ is the Euler totient function.

Now let $p$ be a prime dividing $c, p^{t} \| c$ say. Then $p \mid \Phi_{d}(1)$ by (4.4) which implies that $d=p^{k}, k \geqq 1$, and $\Phi_{d}(1)=p$. Comparing powers of $p$ in both sides of (4.4), we have

$$
t \phi(m) \leqq \phi(m) / \phi(d)
$$

This forces $t=1$ and $\phi(d)=1$. Since $d=p^{k}$, this means that $p=2$ and $k=1$. Hence, $c= \pm 2$ and $\lambda^{2 s+1}=-1$. Substitutimg into (4.3), we obtain

$$
2= \pm 2 \lambda^{s}(1-\lambda)
$$

Squaring this gives $4=-4 \lambda^{-1}(1-\lambda)^{2}$, that is, $\lambda^{2}-\lambda+1=0$, so that $m=$ ord $\lambda=6$. It now follows from $(4.5)$ that $s \equiv 1(\bmod 3), s=1+3 u$ say, and that

$$
\operatorname{sgn} c=\lambda^{1+3 u}(1-\lambda)=\lambda(1-\lambda)(-1)^{u},
$$

that is, $\lambda(\lambda-1)=(-1)^{u+1} \operatorname{sgn} c$. Thus $(-1)^{u}=\operatorname{sgn} c$. Finally, $\lambda^{2 s}=\lambda^{r-1}$, whence $r-1 \equiv$ $2 s \equiv 2(\bmod 6)$. This completes the verification of the conditions of case (iv).

For our second example, we take the group $K_{n}$ given by $(0.2)$, with associated polynomial

$$
f(t)=t^{2}-t+1=\Phi_{6}(t)
$$

the sixth cyclotomic polynomial; it is interesting to note that this same $f$ is also associated with $F(3,1,2, n)$ in the previous example. As above, let $g(t)=t^{n}-1$ and consider four cases.

Case (i): $n \equiv 0(\bmod 6)$. Here $\Phi_{6} \mid g, \rho=0$ and $K_{n}^{a b}$ is infinite.

Case (ii): $n \equiv \pm 1(\bmod 6)$. Here, $K_{n}^{a b}$ is trivial, and $K_{n}$ is perfect.

Case (iii): $n \equiv 3(\bmod 6)$. Here $\rho=4$ and $f$ is irreducible over $\mathbb{F}_{2}$. Its zeros thus belong to $\mathbb{F}_{4} \backslash \mathbb{F}_{2}$ and so have order 3 . Thus they are both zeros of $t^{n}-1$ in this case. Hence, the highest common factor of $\bar{f}$ and $\bar{g}$ has degree 2 and $K_{n}^{a b}$ is non-cyclic, namely $C_{2} \oplus C_{2}$.

Case (iv): $n \equiv+2(\bmod 6)$. Here $\rho=3$ and, modulo $p=3, \bar{f}=(t+1)^{2}$. Since -1 is a simple zero of $\bar{g}$ in this case, it follows that $(\bar{f}, \bar{g})=t+1$, proving that $K_{n}^{a b}$ is cyclic, namely $C_{3}$.

\section{REFERENCES}

1. M. W. Bunder, D. L. Johnson and A.-C. KIM, The exponents of Fibonacci algebras, Fibonacci Quarterly, submitted. 
2. C. M. Camprell and E. F. Robertson, The orders of certain metacyclic groups, Bull. London Math. Soc. 6 (1974), 312-314.

3. J. H. Conway, Advanced problem 5327, Amer. Math. Monthly 72 (1965), 915.

4. J. H. Conway, Solution to advanced problem 5327, Amer. Math Monthly 74 (1967), 91-93.

5. C. W. CURTIS and I. ReINER, Representation theory of finite groups and associative algebras (John Wiley, New York 1962).

6. D. L. Johnson, A note on the Fibonacci groups, Israel J. Math. 17 (1974), 27-282.

7. D. L. Johnson and A.-C. KIM, Cyclic Fibonacci algebras, in preparation.

8. R. M. Thомas, The Fibonacci groups revisited, in Groups St Andrews 1989 (eds. C. M. Campbell and E. F. Robertson), CUP, Cambridge 1991, 445-454.

9. R. M. Thомаs, On a question of Kim concerning certain group presentations, Bull. Korean Math. Soc. 28 (1991), 219-224.

Mathematics Department

UNIVERSITY OF NOTTINGHAM

UnIVERSITY PARK

NotTINGHAM NG7 2RD
Mathematics Department

UNIVERSITY OF GLASGOW

UNIVERSITY GaRdenS

GlasGow G12 8QW 